\title{
Reducts and Modal Operators on Residuated Lattices
}

\author{
William Young \\ Vanderbilt University, Nashville, Tennessee, USA \\ william.j.young@vanderbilt.edu
}

The following are a few standard examples of modal operators on residuated lattices.

Proposition 1. We have the following:

(1) The class of images of Boolean algebras with conuclei is precisely the variety of Heyting algebras.

(2) The class of images of Abelian $\ell$-groups with conuclei is precisely the variety of commutative, cancellative residuated lattices.

(3) The class of images of negative cones of $\ell$-groups with nuclei is precisely the variety of integral GMV -algebras.

In all of these cases, there is a categorical equivalence that bares significant resemblance to certain free objects with respect to forgetful functors. The purpose of this talk is to explore the connections between these modal image functors and their corresponding forgetful functors.

A conucleus on a residuated lattice $\mathbf{A}$ is a map $\sigma: \mathbf{A} \rightarrow \mathbf{A}$ such that $\sigma$ is order-preserving, $\sigma(x) \leq x, \sigma(\sigma(x))=\sigma(x), \sigma(1)=1$ and $\sigma(x) \sigma(y) \leq \sigma(x y)$, for all $x, y \in A$. It is known that the image of a conucleus has a residuated structure.

Proposition 2. If $\mathbf{A}=\langle A, \wedge, \vee, \cdot, \backslash, /, 1, \sigma\rangle$ is a residuated lattice with a conucleus $\sigma$, then the algebra $\mathbf{A}_{\sigma}=\left\langle A_{\sigma}, \wedge_{\sigma}, \vee, \cdot, \backslash_{\sigma}, / \sigma, 1\right\rangle$ is a residuated lattice, where $A_{\sigma}=\sigma[A]$ and for all $x, y \in A_{\sigma}$, $x \wedge_{\sigma} y=\sigma(x \wedge y), x \backslash_{\sigma} y=\sigma(x \backslash y)$, and $x / \sigma y=\sigma(x / y)$.

It will be important to observe that $\mathbf{A}_{\sigma}$ is a $\{\vee, \cdot, 1\}$-subreduct of $\mathbf{A}$. Also, there is a functor which sends residuated lattices with conuclei to their images and maps between such objects to their restrictions on these images.

A nucleus on a residuated lattice $\mathbf{A}$ is a map $\gamma: \mathbf{A} \rightarrow \mathbf{A}$ such that $\gamma$ is order-preserving, $x \leq \gamma(x), \gamma(\gamma(x))=\gamma(x)$, and $\gamma(x) \gamma(y) \leq \gamma(x y)$, for all $x, y \in A$. In the following proposition, note that $\mathbf{A}_{\gamma}$ is a $\{\wedge, \backslash, /\}$-subreduct of $\mathbf{A}$. As with the conuclei, there is a nuclear image functor.

Proposition 3. If $\mathbf{A}=\langle A, \wedge, \vee, \cdot, \backslash, /, 1, \gamma\rangle$ is a residuated lattice with a nucleus $\gamma$, then the algebra $\mathbf{A}_{\gamma}=\left\langle A_{\gamma}, \wedge, \vee_{\gamma},{ }_{\gamma}, \backslash, /, \gamma(1)\right\rangle$ is a residuated lattice, where $A_{\gamma}=\gamma[A]$ and for all $x, y \in$ $A_{\gamma}, x \vee_{\gamma} y=\gamma(x \vee y)$ and $x \cdot \gamma y=\gamma(x \cdot y)$.

For a variety $\mathcal{V}$ of residuated lattices with modal operators, consider $\mathcal{V}^{*}$, the full subcategory of $\mathcal{V}$ consisting of those pairs $\langle\mathbf{B}, \epsilon\rangle$ such that $\mathbf{B}_{\epsilon}$ generates $\mathbf{B}$ as a residuated lattice. 
Theorem 4. We have the following:

(1) There is a categorical equivalence between $\mathcal{B A}_{\sigma}^{*}$ and $\mathcal{H} \mathcal{A}$.

(2) There is a categorical equivalence between $\mathcal{A}_{\sigma}^{*}$ and $\mathcal{C C} a n \mathcal{R} \mathcal{L}$.

(3) There is a categorical equivalence between $\left(\mathcal{L G}_{\gamma}^{-}\right)^{*}$ and $\mathcal{I G M V}$.

In all three of these cases, the free object is constructed by first constructing a free object with respect to a forgetful functor, and then defining a modal operator that recovers the original structure. The next lemma states what the subreducts are in these cases, where $\mathcal{J C M}$ is the class of $\vee$-semilattice-ordered, commutative, cancellative monoids that satisfy $x(y \vee z) \approx x y \vee x z$.

Lemma 5. We have the following:

(1) The class of $\{\wedge, \vee, 0,1\}$-subreducts of Boolean algebras is precisely the class of bounded, distributive lattices.

(2) The class of $\{\vee, \cdot, 1\}$-subreducts of Abelian $\ell$-groups is precisely $\mathcal{J C M}$.

(3) The class of $\{\backslash, /, 1\}$-subreducts of negative cones of $\ell$-groups is precisely the class of cone algebras.

The figure below shows the relationship between the free objects with respect to the modal image functor $\Gamma$ and the forgetful functor $G$. Given a Heyting algebra $\mathbf{A}$, we wish to construct $\Sigma(\mathbf{A})$ such that $\Gamma(\Sigma(\mathbf{A}))=\mathbf{A}$ and $H(\Sigma(\mathbf{A}))=F(G(\mathbf{A}))$. A similar figure holds in the other cases.

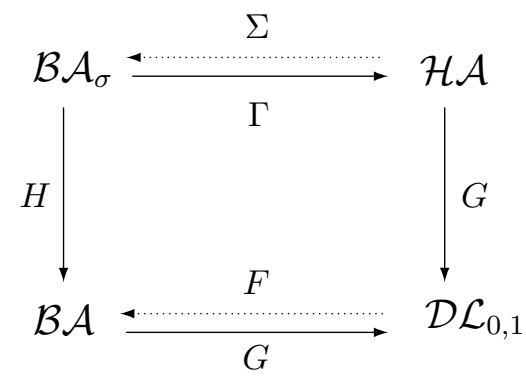

The reason that such nice results hold in these cases is that more can be said about these subreducts. While there is always a free object $F(\mathbf{A})$ with respect to any forgetful functor, in these examples, any time $\mathbf{A}$ is embedded into an algebra $\mathbf{B}$, the subalgebra generated by $A$ in $\mathbf{B}$ is precisely this free object $F(\mathbf{A})$. This explains the use of the term minimal Boolean extension of a bounded, distributive lattice that is often used; there is only one way to extend the operations to get a Boolean algebra. The following definitions are the key to this property.

Definition 6. A set $T$ of terms (in the signature $\tau$ ) is called representative with respect to a subvariety $\mathcal{W}^{\prime}$ of $\mathcal{W}$ if for every algebra $\mathbf{A}$ in $\mathcal{W}^{\prime}$, every element of $F(\mathbf{A})$ can be represented by $t\left(a_{1}, \ldots, a_{n}\right)$, for some term $t \in T$ and some $a_{1}, \ldots, a_{n} \in A$, where we are using a to stand for both the element in $A$ as well as its equivalence class in $F(\mathbf{A})$. 
Obviously, for any subvariety $\mathcal{W}^{\prime}$, the set of all terms in the signature $\tau$ is the largest representative set. As a more interesting example, in the situation of commutative groups and commutative, cancellative monoids, the set $\left\{x^{-1} y\right\}$ is a representative set.

Definition 7. A pair of terms $\left(t_{1}, t_{2}\right)$ (in the signature $\tau$ and with no common variables) is called $\tau^{\prime}$ - reducible if there exists pairs of terms $\left(s_{1}^{i}, s_{2}^{i}\right)$ (in the signature $\tau^{\prime}$ and with variables among the variables in $t_{1}$ and $\left.t_{2}\right)$ such that for every algebra $\mathbf{A}$ in $\mathcal{V}$ and $a_{1}, \ldots, a_{n}, b_{1}, \ldots, b_{m} \in A$, $t_{1}\left(a_{1}, \ldots, a_{n}\right)=t_{2}\left(b_{1}, \ldots, b_{m}\right)$ iff for every $i, s_{1}^{i}\left(a_{1}, \ldots, a_{n}, b_{1}, \ldots, b_{m}\right)=s_{2}^{i}\left(a_{1}, \ldots, a_{n}, b_{1}, \ldots, b_{m}\right)$.

Referring back to the commutative group case, the pair $\left(x^{-1} y, z^{-1} w\right)$ is monoid-reducible (by the pair $(y z, x w)$ ) since for every commutative group $\mathbf{G}$ and $a, b, c, d \in G, a^{-1} b=c^{-1} d$ iff $b c=a d$.

Lemma 8. If there exists a set of terms $T$ that is representative with respect to a subvariety $\mathcal{W}^{\prime}$ of $\mathcal{W}$ and every pair $\left(t_{1}, t_{2}\right)$ of terms of $T$ (where if $t_{1}=t_{2}$, they are taken to have no common variables) is $\tau^{\prime}$-reducible, then whenever an algebra $\mathbf{A}$ in $\mathcal{W}^{\prime}$ can be embedded into an algebra $G(\mathbf{B})$, where $\mathbf{B}$ is in $\mathcal{V}$, the subalgebra of $\mathbf{B}$ generated by the image of $\mathbf{A}$ is (isomorphic to) the free object $F(\mathbf{A})$.

The set $\left\{x^{-1} y\right\}$ works in the case of Abelian $\ell$-groups.

Lemma 9. Let $\mathbf{L}$ be a bounded distributive lattice that is a $\{\wedge, \vee, 0,1\}$-subreduct of the Boolean algebra $\mathbf{B}$. Let $\overline{\mathbf{L}}$ be the Boolean subalgebra of $\mathbf{B}$ generated by L. Then, we have the following:

(1) Every element of $\overline{\mathbf{L}}$ can be written in the form $\bigwedge_{i=1}^{n}\left(a_{i} \vee b_{i}{ }^{\prime}\right)$, where $n$ is a natural number and for $i=1, \ldots, n, a_{i}, b_{i} \in L$, and $x^{\prime}$ denotes the complement of $x$.

(2) Let $n$ and $m$ be natural numbers, and let $a_{i}, b_{i}, c_{j}, d_{j} \in L$, for $i=1, \ldots, n$ and $j=1, \ldots, m$. Let $I=\{1, \ldots, n\}$ and $J=\{1,2\}$. Then, $\bigwedge_{i=1}^{n}\left(a_{i} \vee b_{i}{ }^{\prime}\right) \leq \bigwedge_{j=1}^{m}\left(c_{j} \vee d_{j}{ }^{\prime}\right)$ (in $\left.\mathbf{B}\right)$ iff for every $j=1, \ldots, m$ and every $f \in J^{I}, d_{j} \wedge \bigwedge_{i \in f^{-1}(1)} a_{i} \leq c_{j} \vee \bigvee_{i \in f^{-1}(2)}^{j} b_{i}($ in $\mathbf{L})$.

The situation is a little more complicated in the cone algebras case.

Lemma 10. If a cone algebra $\mathbf{C}$ generates a negative cone of an $\ell$-group $\mathbf{A}$, then every element of $A$ has the form $a_{1} \cdots a_{n}$, for some $a_{1}, \ldots, a_{n} \in C$.

Lemma 11. For every pair $(n, m)$ of positive integers, there exist $m$ cone algebra terms $f_{1}^{n, m}, \ldots, f_{m}^{n, m}$ (in $n+m$ variables) such that for every $\mathbf{A} \in \mathcal{L G}^{-}$and $a_{1}, \ldots, a_{n}, b_{1}, \ldots, b_{m} \in A$, $a_{1} \cdots a_{n} \backslash b_{1} \cdots b_{m}=f_{1}^{n, m}\left(a_{1}, \ldots, a_{n}, b_{1}, \ldots, b_{m}\right) \cdots f_{m}^{n, m}\left(a_{1}, \ldots, a_{n}, b_{1}, \ldots, b_{m}\right)$.

Lemma 12. Let $\mathbf{A} \in \mathcal{L G}^{-}$. Then, $a_{1} \cdots a_{n} \leq b_{1} \cdots b_{m}$ iff each $f_{i}^{n, m}\left(a_{1}, \ldots, a_{n}, b_{1}, \ldots, b_{m}\right)=1$, for $i=1, \ldots, m$.

These representability terms play a key role in establishing the aforementioned categorical equivalences. While these equivalences were already known, this talk will stress the role that the forgetful functors play. The following theorem can also be obtained using this approach. 
Theorem 13. We have the following:

1. Let $\mathcal{W}$ be a subvariety of $\mathcal{H} \mathcal{A}$. Then, there is an interval in the subvariety lattice of $\mathcal{B A}_{\sigma}$ that corresponds to $\mathcal{W}$.

2. Let $\mathcal{W}$ be a subvariety of $\mathcal{C C}$ an $\mathcal{R} \mathcal{L}$. Then, there is an interval in the subvariety lattice of $\mathcal{A}_{\sigma}$ that corresponds to $\mathcal{W}$.

3. Let $\mathcal{W}$ be a subvariety of $\mathcal{I G M V}$. Then, there is an interval in the subvariety lattice of $\mathcal{L G}_{\gamma}^{-}$that corresponds to $\mathcal{W}$.

Now, consider the following recursive definition for the translation $T$ of formulas in the language of residuated lattices to formulas in the language of residuated lattices with a conucleus. Firstly, $T(1)=1$, and for a variable $x, T(x)=\sigma(x)$. For $\circ \in\{\vee, \cdot\}, T(\alpha \circ \beta)=T(\alpha) \circ T(\beta)$, and for $* \in\{\wedge, \backslash, /\}, T(\alpha * \beta)=\sigma(T(\alpha) * T(\beta))$. From the definition of the operations in $\mathbf{A}_{\sigma}$, it is fairly easy to see that for any formula $\phi$ in the language of residuated lattices, $\mathbf{A}_{\sigma}=\phi$ iff $\langle\mathbf{A}, \sigma\rangle \models T(\phi)$. A similar translation $D$ exists for nuclei on residuated lattices such that $\mathbf{A}_{\gamma} \models \phi$ iff $\langle\mathbf{A}, \gamma\rangle \models D(\phi)$.

Proposition 14. We have the following:

1. Let $\mathcal{W}$ be a subvariety of $\mathcal{H} \mathcal{A}$ and $\mathcal{V}$ be a subvariety of $\mathcal{B} \mathcal{A}_{\sigma}$. Then, $\mathcal{V}$ is in the interval that corresponds to $\mathcal{W}$ if and only if $\mathcal{V}=T(\phi)$ exactly when $\mathcal{W} \models \phi$.

2. Let $\mathcal{W}$ be a subvariety of $\mathcal{C C}$ an $\mathcal{R} \mathcal{L}$ and $\mathcal{V}$ be a subvariety of $\mathcal{A}_{\sigma}$. Then, $\mathcal{V}$ is in the interval that corresponds to $\mathcal{W}$ if and only if $\mathcal{V}=T(\phi)$ exactly when $\mathcal{W} \models \phi$.


that corresponds to $\mathcal{W}$ if and only if $\mathcal{V} \models D(\phi)$ exactly when $\mathcal{W} \models \phi$.

While the first of these was already known, the proofs presented here are different from those originally done, since most of the work is shifted to the reducts. This allows for a deeper understanding of why there is such similarity in these three cases. 